

18 a 21 de novembro de 2014, Caldas Novas - Goiás

\title{
IMPROVED SIMULATED ANNEALING
}

\author{
Vinícius Nunes Carvalho, viniciusncarvalho92@gmail.com ${ }^{1}$ \\ Sezimária de Fátima Pereira Saramago, saramago@ufu.br²
}

\author{
${ }^{1}$ Universidade Federal de Uberlândia - Faculdade de Engenharia Mecânica, Av. João Naves de Ávila, no 2160 , \\ Uberlândia, Minas Gerais, Brasil. \\ ${ }^{2}$ Universidade Federal de Uberlândia - Faculdade de Matemática, Av. João Naves de Ávila, no 2160, Uberlândia, \\ Minas Gerais, Brasil.
}

\begin{abstract}
Resumo: $O$ principal objetivo deste trabalho é aprimorar o algoritmo conhecido como Recozimento Simulado (Simulated Annealing), visando aproveitar suas potencialidades e aumentar sua eficiência para a solução de problemas de otimização. Na pesquisa pretende-se modificar o algoritmo básico de Simulated Annealing desenvolvendo o método denominado Improved Simulated Annealing (ISA). O algoritmo original é serial na sua concepção e requer elevado tempo computacional. Cada ciclo do algoritmo é composto pela geração, avaliação da função objetivo e aplicação de critérios de decisão, sendo necessárias várias iterações para cada valor de temperatura, somente ao final deste ciclo o valor do ponto ótimo é atualizado. Os parâmetros do algoritmo são: a função custo, que representa a energia do sistema; as variáveis de projeto, que descrevem sua configuração e a temperatura, que é um parâmetro de controle. Se o parâmetro temperatura $(T)$ tiver magnitude muito superior ao desvio padrão da função no intervalo, quase todos os pontos são aceitos. Ao passo que se T for igual a zero, o método se torna uma busca aleatória do mínimo. Assim, adota-se: $T_{i}$ como o valor do desvio padrão da função objetivo no intervalo estudado e $T_{f}$ com a ordem de grandeza desejada para a precisão do ponto ótimo. Com este estudo pretende-se que a eficiência do método seja melhorada por meio das modificações pontuais feitas no programa visando o melhor aproveitamento de sua capacidade para as variáveis envolvidas no projeto. Além disso, o algoritmo será adaptado para trabalhar com funções multi-objetivo na presença de restrições. O algoritmo desenvolvido é aplicado no projeto ótimo de uma caixa de engrenagens e os resultados apresentados para demonstrar a eficiência da metodologia proposta.
\end{abstract}

Palavras-chave: Otimização, Simulated Annealing, Improved Simulated Annealing, Caixa de Engrenagem.

\section{INTRODUÇÃO}

Otimização matemática é um ramo da ciência computacional que busca a melhor solução para um dado problema, procura projetar o novo com a máxima eficiência e consequentemente com o menor custo. Neste método a intenção é obter a solução ótima sem que haja perda de tempo, ou seja, testando um número relativamente pequeno de possibilidades e ainda assim com sucesso. Em geral, problemas de otimização em engenharia são caracterizados por um grande número de restrições, normalmente modelados por funções numéricas de várias variáveis, sendo que o objetivo pode ser pontos de máximo ou de mínimo destas funções, variando em cada aplicação. Estes problemas surgem em todas as áreas do conhecimento variando apenas a formulação dos mesmos, sendo bastante grande a gama de técnicas disponíveis para resolvê-los.

Com o aumento das restrições e o número de variáveis do projeto o problema fica mais complexo e deste modo há o aumento do custo computacional para obter uma solução. Quando as funções são descontínuas, ou funções com presença de muitos mínimos locais, torna-se mais difícil a obtenção do mínimo global. O avanço dos recursos computacionais ocorridos nos últimos anos, e o fato de que os problemas tornam-se cada vez mais complexos, tem favorecido o avanço das técnicas de otimização heurísticas. O Simulated Annealing pertence à classe de métodos que tentam simular os processos usados pela natureza para resolver problemas complexos de otimização. Segundo Faria e Saramago (2001), nesta técnica a analogia utilizada é com o crescimento de um cristal simples de um metal fundido, que corresponde a encontrar o mínimo local da energia interna do metal (função objetivo), sendo que esta é uma função da disposição dos átomos (variáveis de projeto). A motivação deste trabalho é melhorar a eficiência deste método de otimização, visando um custo computacional menor, desenvolvendo um novo algoritmo chamado Improved Simulated Annealing. Foram realizadas algumas modificações no algoritmo já existente para que a convergência do método fosse melhorada, além de considerar um novo critério de parada para controlar a estagnação do algoritmo evitando iterações desnecessárias. 


\section{MÉTODO DE OTIMIZAÇÃO}

\subsection{Forma Geral de um Método de Otimização}

O problema geral de otimização consiste em minimizar uma função objetivo, sujeita, ou não, a restrições de igualdade, desigualdade e restrições laterais. A função objetivo e as funções de restrições podem ser funções lineares ou não lineares em relação às variáveis de projeto, implícitas ou explícitas, calculadas por técnicas analíticas ou numéricas.

Seja o problema geral de otimização dado por:

$$
\begin{aligned}
& \text { Minimizar } F(x)=\left[\begin{array}{lllll}
F_{1}(x) & F_{2}(x) & \ldots & F_{k}(x)
\end{array}\right]^{T}, \quad F(x) \in E^{k} \text {; } \\
& x=\left[x_{1}, x_{2}, \ldots, x_{n}\right]^{T}, x \in R \text {. } \\
& g_{j}(x) \geq 0, j=1,2, \ldots, J \text {; } \\
& \text { Sujeito a: } \quad h_{l}(x)=0, l=1,2, \ldots, L \text {; } \\
& x_{i}^{(L)} \leq x \leq x_{i}^{(U)}, i=1,2, \ldots, n \text {. }
\end{aligned}
$$

sendo que $F(x)$ representa o vetor das funções objetivo, $g_{j}$ e $h_{k}$ são, respectivamente, as restrições de desigualdade e de igualdade, $\mathrm{x}_{i}^{(L)}$ e $x_{i}^{(U)}$ são os limites inferiores e superiores de cada variável de projeto, $E^{k}$ o espaço euclidiano e $R$ o conjunto dos números reais.

\subsection{Simulated Annealing}

Simulated Annealing pertence à mesma classe dos métodos das Redes Neurais e Algoritmos Genéticos, no sentido que simulam métodos Naturais. O algoritmo Simulated Annealing permite uma útil conexão entre a mecânica estatística (comportamento de um sistema de vários graus de liberdade em equilíbrio térmico a uma temperatura finita) e a otimização combinatória (encontrar um mínimo de uma dada função dependendo de vários parâmetros).

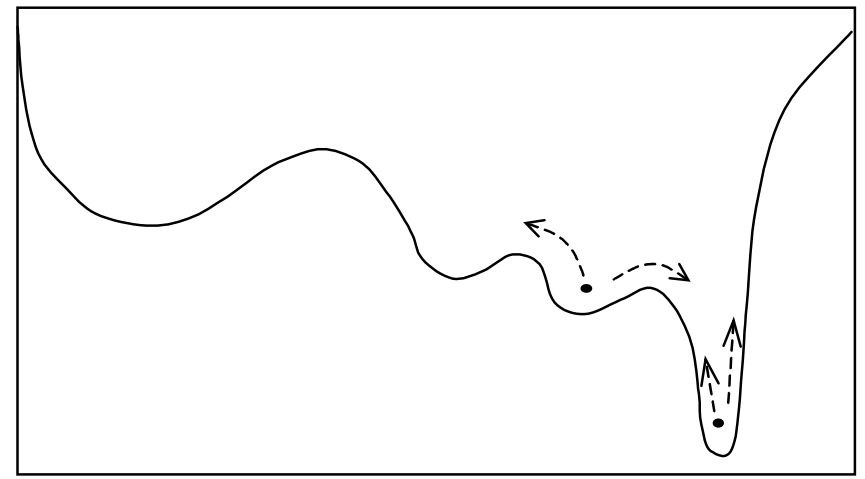

Figura 1. Analogia entre o processo de otimização e o recozimento simulado. Fonte: Saramago (2003).

Metropolis et al (1953) introduziram um método numérico simples que representa o estado equilíbrio de um conjunto de átomos a uma dada temperatura. A analogia com o processo de otimização pode ser feita analisando a Fig. 1. Este método de otimização faz uma analogia com o processo de recozimento (annealing) da metalurgia. Sabe-se da Metalurgia que, se o metal é resfriado em condições apropriadas, o cristal simples pode ser obtido (Kirkpatrick, 1983).

No recozimento o metal é aquecido a altas temperaturas, causando um choque violento nos átomos. Se o metal for resfriado de forma brusca, a microestrutura tende a um estado randomicamente instável, porém, se o metal é resfriado de forma suficientemente lenta, o sistema procurará um ponto de equilíbrio caracterizado por uma microestrutura ordenada e estável. As variáveis de projeto são perturbadas randomicamente e armazena-se o melhor valor da função objetivo a cada perturbação. A temperatura é então reduzida (annealing) e novas tentativas executadas. Este procedimento continua até escaparmos de um mínimo local. Ao final do processo é possível que se obtenha um mínimo global.

Seja $\Delta \mathrm{E}$ a energia de um sistema de átomos a uma temperatura $\mathrm{T}$. Em cada passo do algoritmo, é dado um deslocamento aleatório a um átomo, o que implica uma nova energia do sistema $\Delta \mathrm{E}$. Se esta nova energia $\Delta \mathrm{E}$ é menor ou igual a zero $(\Delta \mathrm{E} \leq 0)$, o deslocamento é aceito, caso contrário $(\Delta \mathrm{E}>0)$, a probabilidade da configuração ser aceita será dada pela equação:

$$
P(\Delta E)=e^{\left(-\Delta E / K_{b} T\right)} \text {, sendo que } K_{b} \text { é a constante de Boltzmann. }
$$


Um número aleatório $r$, uniformemente distribuído, deve ser gerado no intervalo [0, 1]. Se $r \leq P(\Delta E)$ a nova configuração é aceita. Se $r>P(\Delta E)$ a configuração anterior é utilizada para iniciar um novo passo.

A escolha da função de probabilidade $P(\Delta E)$, conforme acima descrito, se deve ao fato de que o sistema evolui segundo uma distribuição de Boltzman.

Os parâmetros do algoritmo são: a função custo, que representa a energia do sistema; as variáveis de projeto, que descrevem sua configuração e a temperatura, que é um parâmetro de controle. A Fig. 2 apresenta o fluxograma desta metodologia.

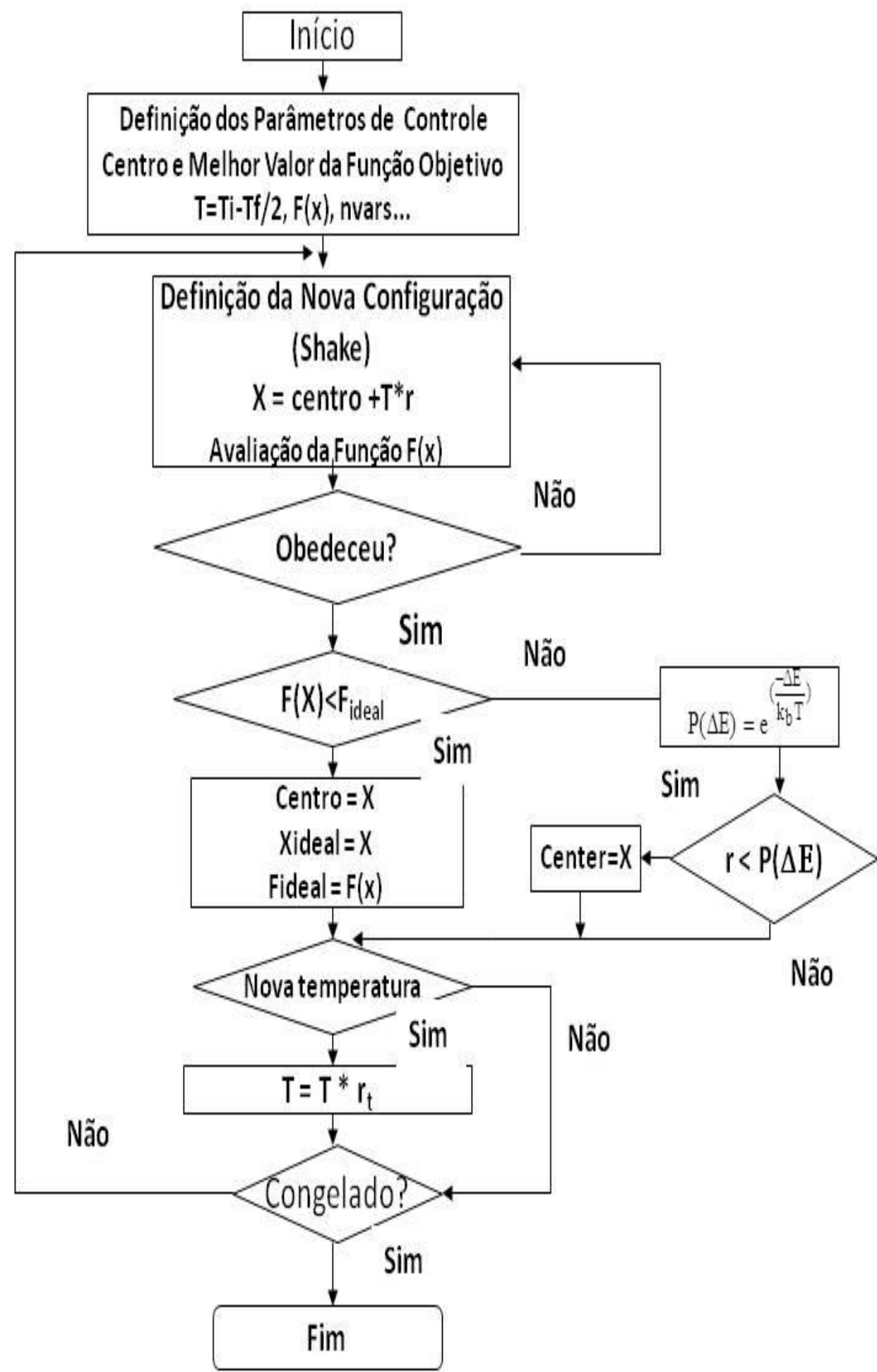

Figura 2 - Fluxograma para o algoritmo Simulated Annealing. 
Se $T$ tiver magnitude muito superior ao desvio padrão da função no intervalo, quase todos os pontos são aceitos. Ao passo que se $T$ for igual a zero, o método se torna uma busca aleatória do mínimo. Assim, adota-se: $T_{i}$ como o valor do desvio padrão da função objetivo no intervalo estudado e $T_{f}$ com a ordem de grandeza desejada para o precisão do ponto ótimo.

Na otimização via Simulated Annealing (Saramago e Faria, 2001) considera-se: a perturbação randômica das variáveis de projeto e a manutenção do melhor valor da função objetivo.

Após algumas tentativas o melhor valor da função é chamado de centro, em torno do qual ocorreram as perturbações na próxima temperatura. Os parâmetros de controle para iniciar o procedimento são: a função objetivo $F(x)$, as variáveis de projeto iniciais $(x)$, o número de variáveis de projeto, a temperatura inicial $T_{i}$, a temperatura final $T_{f}$, o número de iterações para cada temperatura, o número de temperaturas $n_{\text {temp }}$, o número de avaliações da função objetivo, o critério de parada.

A configuração inicial das variáveis de decisão é adotada como centro. O valor inicial da função objetivo adotado como o melhor valor (best fucntion value).

No próximo passo, o número aleatório $r$ é gerado e as variáveis são modificadas (shake):

$$
r=r_{1}+r_{2}-r_{3}-r_{4}
$$

e

$$
T=T \frac{\sqrt{12}}{12} ; x=\text { center }+T * r
$$

$\mathrm{Na}$ Eq. (4) observa-se que quatro valores aleatórios são gerados, de forma que a variável $r$ seja adotada como média zero (Masters, 1993). Assim, uma nova configuração é obtida pela Eq. (5) e um novo valor da função objetivo pode ser calculado. O esquema inicia-se com uma temperatura alta, que é reduzida discretamente, usando o fator $0<$ $r_{t}<1$, até que o sistema "resfrie", conforme as expressões abaixo:

$$
r_{t}=e^{\frac{\ln \left(T_{f} / T_{i}\right)}{n_{\text {temp }}-1}}, \quad T=T * r_{t} .
$$

A temperatura é então reduzida (annealing) e novas tentativas são executadas. Este procedimento continua até escaparmos de um mínimo local. Ao final do processo é possível que se obtenha um mínimo global.

\subsection{Improved Simulated Annealing}

O objetivo desta pesquisa é modificar o algoritmo original visando uma melhor eficácia do método e também buscando um menor custo computacional. O fluxograma do algoritmo modificado pode ser verificado na Fig. 3 . A seguir serão apresentadas as mudanças feitas e suas justificativas.

A primeira alteração proposta foi a utilização de uma temperatura inicial para cada variável de projeto obtida pela média aritmética de suas restrições laterais, ou seja:

$$
T_{i}=\frac{x_{i}^{(L)}+x_{i}^{(U)}}{2} .
$$

sendo que $\mathrm{x}_{i}^{(L)}$ e $x_{i}^{(U)}$ representam as restrições laterais de cada variável.

Observe que no algoritmo anterior era adotada uma temperatura inicial única para todas as variáveis. Como as variáveis podem ter valores muito diferentes entre si, nesta nova proposta, o processo de otimização fica mais eficiente, sendo capaz de atingir um espaço de busca muito maior.

Outra mudança proposta foi no critério de parada do algoritmo. No algoritmo original testava-se se a função objetivo convergia para um valor fixo, pré-estabelecido. Nesta nova proposta é avaliada a diferença dos valores ótimos da função nas duas últimas iterações. Cada vez que o valor obtido for inferior a uma precisão estabelecida, o que significa que nestas duas últimas iterações a função está estagnada, acrescenta-se uma unidade a um contador de controle (control), ou seja:

$$
\left|F(x)_{k+1}-F(x)_{k}\right|<\varepsilon \Rightarrow \text { control=control }+1 \text {. }
$$




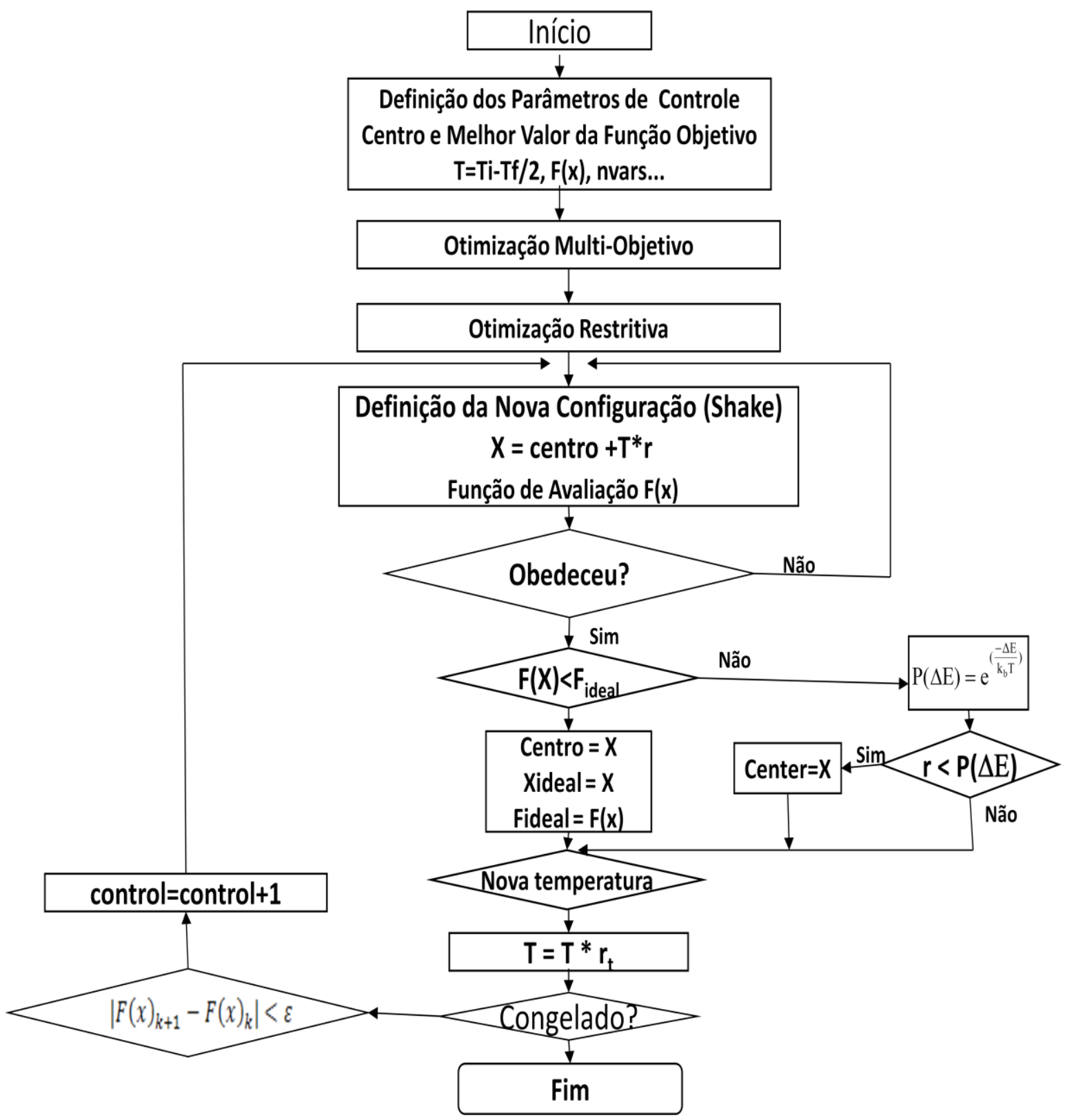

Figura 3 - Fluxograma para o algoritmo Improved Simulated Annealing.

Uma nova adaptação efetuada no algoritmo foi na função "shake", visando garantir que os novos valores adotados para cada variável $x_{i}$ estejam dentro das restrições laterais, assim foi imposto que:

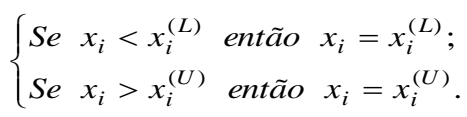

Além disso, o algoritmo foi adaptado para trabalhar com problemas de otimização multi-critérios, substituindo o vetor de funções objetivo por um problema de otimização escalar. Uma das técnicas é utilizar o método da ponderação dos objetivos, através da criação de uma função da forma:

$$
f(x)=\sum_{i=1}^{k} \frac{w_{i} f_{i}(x)}{f_{i}^{0}} .
$$

Seria desejável que os coeficientes de ponderação $w_{k}$ representassem a importância relativa de cada critério. Para que $w_{k}$ possa refletir de forma aproximada a importância dos objetivos, todas as funções devem ser expressas em unidades que aproximem os mesmos valores numéricos. Assim, na Eq.(10), a função vetorial foi normalizada pelo uso 
da solução ideal $f_{k}^{0}$. Esta solução é determinada obtendo-se separadamente o mínimo viável, para todas as funções objetivo. Em outras palavras:

$$
f_{k}^{o}=\min f_{k}(x), \quad k=1, \ldots, K \quad \text { sujeito as restrições (2). }
$$

A função escalar também pode ser determinada por famílias de métricas $L_{p}$, definidas por:

$$
L_{p}(f)=\left[\sum_{i=1}^{k}\left|f_{i}^{o}-f_{i}(x)\right|^{s}\right]^{1 / s}, \quad 1 \leq s \leq \infty .
$$

Muitas vezes ainda é recomendado o uso de distância relativa ao invés de distância absoluta, logo a equação acima pode ser reescrita como:

$$
L_{p}(f)=\left[\sum_{i=1}^{k}\left|\frac{f_{i}^{o}-f_{i}(x)}{f_{i}^{o}}\right|^{s}\right]^{1 / s}, \quad 1 \leq s \leq \infty .
$$

Falta ainda adaptar o algoritmo para trabalhar com problemas na presença de restrições. Neste estudo, foi utilizado o conceito de função de penalidade. Nesta técnica, os problemas com restrições são transformados em problemas sem restrições adicionando uma função de penalidade $P(x)$ à função objetivo original para limitar as violações das restrições:

$$
\begin{aligned}
& \phi(x)=f(x)+r_{p} P(x) ; \\
& P(x)=\left[\sum_{j=1}^{J}\left\{\max \left[o, g_{j}(x)\right]\right\}^{2}+\sum_{l=1}^{L}\left[h_{l}(x)\right]^{2}\right] .
\end{aligned}
$$

onde $f(x)$ é a função objetivo original, $P(x)$ é uma função de penalidade imposta, $g_{j}(x)$ e $h_{l}(x)$ são funções de restrições de desigualdade e igualdade, respectivamente, conforme a Eq.(2). O escalar $r_{p}$ é um multiplicador que quantifica a magnitude da penalidade. Para a eficiência do método, deve-se procurar um valor adequado para o fator de penalidade de forma a garantir que todas as restrições sejam obedecidas.

Para testar as alterações, a seguinte função teste foi utilizada:

$$
F(x)=\sum_{i=1}^{n-1} 100 *\left(x_{i+1}^{2}+x_{i}^{2}\right)+\left(1+x_{i}^{2}\right), n=3 .
$$

Na Fig. 4 podem-se acompanhar os valores da função objetivo dada pela Eq. (15) ao longo das iterações. A Fig. 4a considera valores fixos para a temperatura inicial $T_{i}=0.5$ e a Fig. $4 \mathrm{~b}$ apresenta os resultados obtidos considerando uma temperatura inicial diferente para cada variável, calculada conforme Eq. (7).

Note que a mudança feita no algoritmo garantiu convergência progressiva do programa para a solução ótima, assim confirma-se que a modificação feita resultou em uma melhor eficiência do algoritmo.

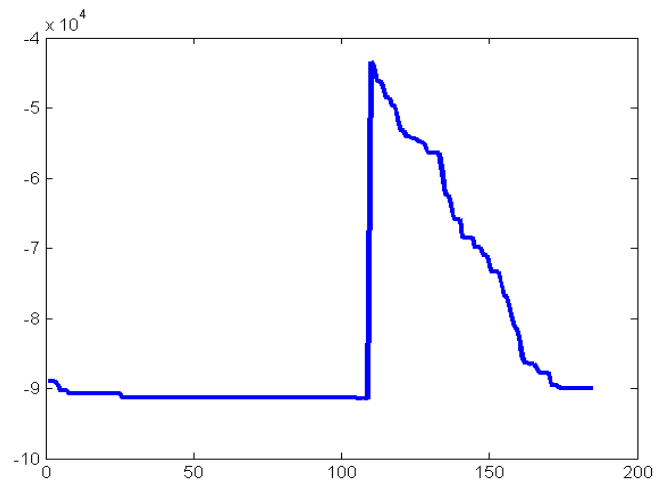

a)

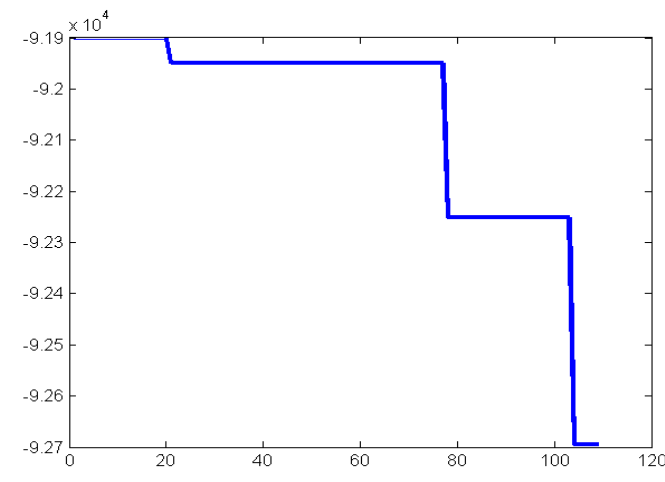

b)

Figura 4. Resultados para a função objetivo ao longo das iterações modificando a temperatura inicial: a) Temperatura inicial igual a 0.5; b) Temperatura inicial dada pela Eq. (7). 
A fim de comparação, a função dada pela Eq. (15) foi otimizada utilizando os dois algoritmos para a modificação do critério de parada mostrado na Eq.(8). Por meio da Fig. 5a e 5b, observa-se que o tempo de convergência foi bem menor no método modificado, deste modo conclui-se que o novo critério de parada foi mais eficiente, diminuindo consideravelmente o tempo computacional. Mesmo com esse menor número de iterações a convergência continuou sendo garantida.



a)

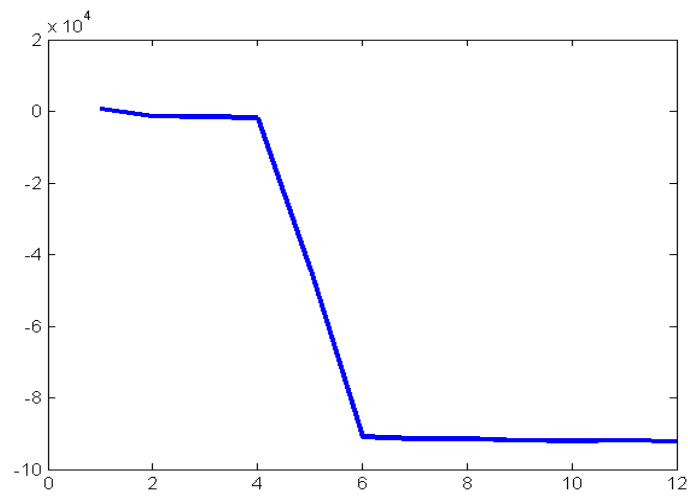

b)

Figura 5. Resultados para a função objetivo ao longo das iterações utilizando novo critério de parada:
a) Simulated Annealing;
b) Improved Simulated Annealing.

\subsection{Aplicação}

Seja o projeto ótimo da caixa de transmissão de um torno mecânico, apresentado em Saramago e Souza (2006), formulado como:

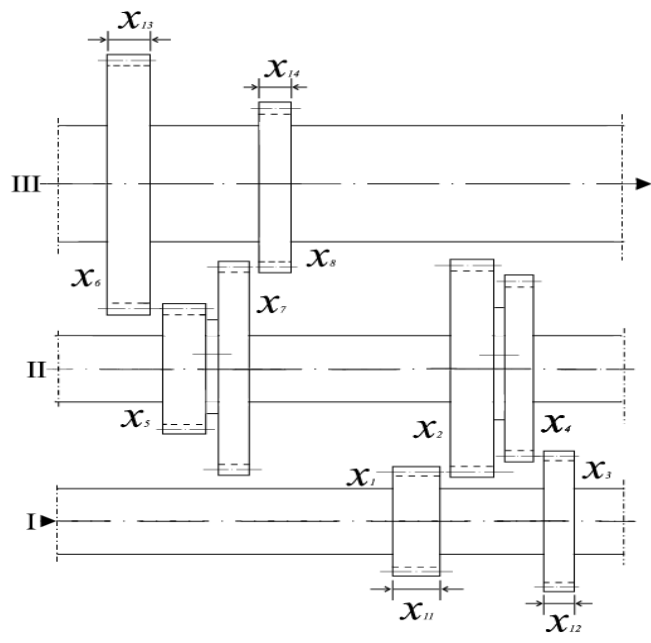

Figura 6. Esquema para a Caixa de engrenagens. Fonte: Saramago e Souza (2006).

Dada a estrutura do torno mecânico, os parâmetros cinemáticos nos eixos de entrada e saída e a força potencial no eixo de entrada, determinar uma solução, obtendo os parâmetros básicos como: número de dentes, módulo e largura dos dentes. De forma a minimizar as seguintes funções objetivo:

i) Volume do material usado para os elementos montados sobre os eixos, $f_{l}(x)$;

ii) Maximizar a velocidade periférica entre as engrenagens, $f_{2}(x)$;

iii) Largura das caixas de transmissão, $f_{3}(x)$;

iv) Distancia entre os eixos de entrada e saída, $f_{4}(x)$.

Além disso, o projeto ótimo deve satisfazer às restrições relacionadas com a geometria, cinemática e tensão. Observe que a segunda função objetivo reflete o comportamento dinâmico da caixa de câmbio (ruídos e vibração) quando opera a altas velocidades. As últimas funções representam o peso e dimensão da caixa de transmissão.

As variáveis de projeto, conforme esquematizado na Fig. 6, são: 
$-x_{1}, x_{2}, x_{3}, x_{4}, x_{5}, x_{6}, x_{7}, x_{8}$ representam o número de dentes da engrenagem;

- $x_{9}$ e $x_{10}$ módulo das engrenagens montadas em conjunto (eixos - duplos);

$-x_{11}, x_{12}, x_{13}, x_{14}$ representam a largura dos dentes da engrenagem.

O projeto cinemático, o desenho estrutural e os dados básicos para esta caixa de transmissão são esquematizados na Fig. 6. O problema do projeto ótimo do torno mecânico foi formulado como:

$$
\begin{gathered}
\min f_{1}(x)=7,86 \times 10^{-17} x_{9}^{2} x_{11}\left(\left(x_{1}+2\right)^{2}+\left(x_{2}+2\right)^{2}\right)+x_{9}^{2} x_{12}\left(\left(x_{3}+2\right)^{2}+\left(x_{4}+2\right)^{2}\right)+ \\
7,86 \times 10^{-17} x_{9}^{2} x_{11} x_{10}^{2} x_{13}\left(\left(x_{5}+2\right)^{2}+\left(x_{6}+2\right)^{2}\right)+7,86 \times 10^{-17} x_{9}^{2} x_{11} x_{10}^{2} x_{14}\left(\left(x_{7}+2\right)^{2}+\left(x_{8}+2\right)^{2}\right) d c m^{3} \\
\max f_{2}(x)=0,0732 \frac{x_{3}}{x_{4}} x_{7} x_{10} \mathrm{~m} / \mathrm{s} ; \\
\min f_{3}(x)=2\left(x_{11}+x_{12}+x_{13}+x_{14}\right) \mathrm{mm} ; \\
\min f_{4}(x)=0,5\left(x_{9}\left(x_{1}+x_{2}\right)+x_{10}\left(x_{5}+x_{6}\right)\right) \mathrm{mm} .
\end{gathered}
$$

Sujeito às seguintes restrições:

$$
\begin{aligned}
& x_{1}-14 \geq 0, x_{2}-14 \geq 0, x_{3}-14 \geq 0 \text {, } \\
& x_{4}-14 \geq 0, x_{5}-14 \geq 0, x_{6}-14 \geq 0 \text {, } \\
& x_{1}+x_{2}-35 \geq 0,100-x_{1}-x_{2} \geq 0 \text {, } \\
& x_{2}+x_{4}-35 \geq 0,100-x_{3}-x_{4} \geq 0 \text {, } \\
& x_{5}+x_{6}-35 \geq 0,100-x_{5}-x_{6} \geq 0 \text {, } \\
& x_{7}+x_{8}-35 \geq 0,100-x_{7}-x_{8} \geq 0, \\
& 2-\frac{x_{1}}{x_{2}} \geq 0,2-\frac{x_{3}}{x_{4}} \geq 0,2-\frac{x_{5}}{x_{6}} \geq 0,2-\frac{x_{7}}{x_{8}} \geq 0, \\
& \frac{x_{1}}{x_{2}}-0,25 \geq 0, \frac{x_{3}}{x_{4}}-0,25 \geq 0, \frac{x_{5}}{x_{6}}-0,25 \geq 0, \frac{x_{7}}{x_{8}}-0,25 \geq 0 \text {, } \\
& 51,12-\frac{280 x_{1} x_{5}}{x_{2} x_{6}} \geq 0, \frac{280 x_{1} x_{5}}{x_{2} x_{6}}-54,88, \\
& 183,6-\frac{280 x_{1} x_{7}}{x_{2} x_{8}} \geq 0, \frac{280 x_{1} x_{7}}{x_{2} x_{8}}-174,4 \geq 0, \\
& 114,24-\frac{280 x_{3} x_{5}}{x_{4} x_{6}} \geq 0, \frac{280 x_{3} x_{5}}{x_{4} x_{6}}-109,76 \geq 0, \\
& 362,1-\frac{280 x_{3} x_{7}}{x_{4} x_{8}} \geq 0, \frac{280 x_{3} x_{7}}{x_{4} x_{8}}-347,9 \geq 0, \\
& x_{9}\left(x_{1}-2,5\right)-46 \geq 0, x_{9}\left(x_{3}-2,5\right)-46 \geq 0 \text {, } \\
& x_{9}\left(x_{2}-2,5\right)-60 \geq 0, x_{9}\left(x_{4}-2,5\right)-60 \geq 0 \text {, } \\
& x_{10}\left(x_{5}-2,5\right)-60 \geq 0, x_{10}\left(x_{7}-2,5\right)-60 \geq 0 \text {, } \\
& x_{10}\left(x_{6}-2,5\right)-100 \geq 0, x_{10}\left(x_{8}-2,5\right)-100 \geq 0 \text {, } \\
& x_{9}\left(x_{1}+x_{2}\right)-x_{10} x_{5}-96 \geq 0, x_{9}\left(x_{1}+x_{2}\right)-x_{10} x_{7}-96 \geq 0 \text {, } \\
& x_{10}\left(x_{5}+x_{6}\right)-x_{9} x_{2}-204 \geq 0, x_{10}\left(x_{5}+x_{6}\right)-x_{4} x_{2}-204 \geq 0 \text {, } \\
& \frac{x_{11}}{x_{9}}-6 \geq 0, \frac{x_{12}}{x_{9}}-6 \geq 0, \frac{x_{13}}{x_{10}}-6 \geq 0, \frac{x_{14}}{x_{10}}-6 \geq 0, \\
& 12-\frac{x_{11}}{x_{9}} \geq 0,12-\frac{x_{12}}{x_{9}} \geq 0,12-\frac{x_{13}}{x_{10}} \geq 0,12-\frac{x_{14}}{x_{10}} \geq 0, \\
& 393-\frac{1240000}{x_{1} x_{9}^{2} x_{11}} \geq 0,393-\frac{1240000}{x_{3} x_{9}^{2} x_{12}} \geq 0, \\
& 393-\frac{6010000}{x_{6} x_{10}^{2} x_{13}} \geq 0,393-\frac{1870000}{x_{8} x_{10}^{2} x_{14}} \geq 0, \\
& 44,2-\frac{2850000}{x_{1}^{2} x_{8}^{2}\left(x_{11}-0,5 x_{8}\right)} \geq 0,44,2-\frac{2850000}{x_{1} x_{2} x_{9}^{2}\left(x_{11}-0,5 x_{8}\right)} \geq 0, \\
& 44,2-\frac{2850000}{x_{3}^{2} x_{8}^{2}\left(x_{12}-0,5 x_{8}\right)} \geq 0,44,2-\frac{2850000}{x_{3} x_{4} x_{8}^{2}\left(x_{11}-0,5 x_{8}\right)} \geq 0, \\
& 44,2-\frac{13700000}{x_{5} x_{6} x_{10}^{2}\left(x_{13}-0,5 x_{10}\right)} \geq 0,44,2-\frac{13700000}{x_{6}^{2} x_{10}^{2} x_{9}^{2}\left(x_{13}-0,5 x_{10}\right)} \geq 0 \text {, } \\
& 44,2-\frac{2850000}{x_{7} x_{8} x_{10}^{2}\left(x_{14}-0,5 x_{10}\right)} \geq 0,44,2-\frac{2850000}{x_{8}^{2} x_{10}^{2}\left(x_{14}-0,5 x_{10}\right)} \geq 0 .
\end{aligned}
$$

Para o problema multi-critério, os seguintes valores ideais, calculados conforme Eq. (11), foram obtidos:

$$
f_{1}^{\mathrm{o}}=1457,4 \mathrm{dcm}^{3} \quad f_{2}{ }^{\mathrm{o}}=-147,4 \mathrm{~m} / \mathrm{s} \quad f_{3}{ }^{\mathrm{o}}=108,6 \mathrm{~mm} \quad f_{4}^{\mathrm{o}}=3964,5 \mathrm{~mm}
$$


Com a otimização do problema foi notado a presença de vários mínimos locais devido aos diferentes resultados apresentados ao se usar técnicas sequenciais. Outro fator importante a ser ressaltado sobre este problema é que existem muitas restrições envolvendo as variáveis de decisão, esta imposição dificulta a otimização. Após várias tentativas, os melhores resultados são apresentados a seguir, representados por $\mathrm{x}_{\text {best }}$ e $\mathrm{f}\left(\mathrm{x}_{\text {best }}\right)$. Para o cálculo das médias e dos respectivos desvios padrão, o programa foi executado 10 vezes, e os valores obtidos apresentados como $x_{\text {med }}$ e $f\left(x_{\text {med }}\right)$.

Com a aplicação do Improved Simulated Annealing, trabalhando com a função de ponderação dos objetivos definida na Eq. (10), considerando todos os coeficientes de ponderação iguais $\left(w_{i}=0,25\right)$, obteve-se os resultados apresentados abaixo:

$$
\begin{aligned}
& \min f(x)=0,25 \frac{f_{1}}{f_{1}^{0}}+0,25 \frac{f_{2}}{f_{2}^{0}}+0,25 \frac{f_{3}}{f_{3}^{0}}+0,25 \frac{f_{4}}{f_{4}^{0}}
\end{aligned}
$$

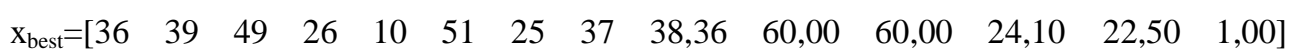

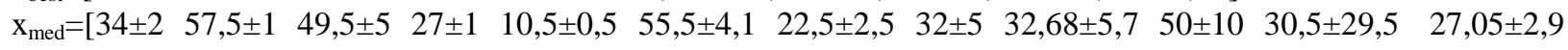

$$
\begin{aligned}
& 15,25 \pm 7,2513 \pm 12] \\
& \mathrm{f}\left(\mathrm{x}_{\text {best }}\right)=\left[\begin{array}{llll}
505,51 \mathrm{dcm}^{3} \quad 206,93 \mathrm{~m} / \mathrm{s} \quad 215,20 \mathrm{~mm} \quad 3268,4 \mathrm{~mm}
\end{array}\right] \\
& f\left(x_{\text {med }}\right)=\left[1003,85 \pm 498,3 \mathrm{dcm}^{3} \quad 186,45 \pm 20,47 \mathrm{~m} / \mathrm{s} \quad 267,62 \pm 5,45 \mathrm{~mm} \quad 3625,55 \pm 357,1 \mathrm{~mm}\right]
\end{aligned}
$$

Para a métrica $\mathrm{L}_{2}$, obtida a partir da Eq. (12), os resultados calculados por meio do ISA, foram:

$$
\begin{aligned}
& \min f(x)=\left[\left(f_{1}^{0}-f_{1}\right)^{2}+\left(f_{2}^{0}-f_{2}\right)^{2}+\left(f_{3}^{0}-f_{3}\right)^{2}+\left(f_{4}^{0}-f_{4}\right)^{2}\right]^{1 / 2} .
\end{aligned}
$$

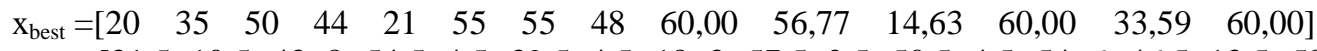

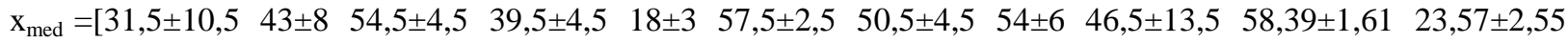

$$
\begin{aligned}
& 36,85 \pm 21,7 \quad 30,85 \pm 7 \quad 43,85 \pm 16,15] \\
& \mathrm{f}\left(\mathrm{x}_{\text {best }}\right)=\left[2090,4 \mathrm{dcm}^{3} \quad 259,72 \mathrm{~m} / \mathrm{s} \quad 336,44 \mathrm{~mm} \quad 3807,3 \mathrm{~mm}\right] \\
& f\left(x_{\text {med }}\right)=\left[\begin{array}{lll}
\left.1547,2 \pm 543 \mathrm{dcm}^{3} \quad 282,67 \pm 22,9 \mathrm{~m} / \mathrm{s} \quad 270,36 \pm 66,1 \mathrm{~mm} \quad 3785,45 \pm 21,85 \mathrm{~mm}\right]
\end{array}\right.
\end{aligned}
$$

E por fim, os valores ótimos calculados por meio do ISA, usando uma métrica relativa $\mathrm{L}_{3}$, conforme definido na Eq. (13), podem ser observados abaixo:

$$
\begin{aligned}
& \min f(x)=\left(\left|\frac{f_{1}^{0}-f_{1}}{f_{1}^{0}}\right|^{3}+\left|\frac{f_{2}^{0}-f_{2}}{f_{2}^{0}}\right|^{3}+\left|\frac{f_{3}^{0}-f_{3}}{f_{3}^{0}}\right|^{3}+\left|\frac{f_{4}^{0}-f_{4}}{f_{4}^{0}}\right|^{3}\right)^{1 / 3} .
\end{aligned}
$$

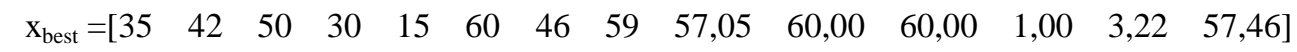

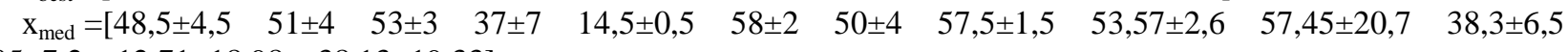

$$
\begin{aligned}
& 8,05 \pm 7,2 \quad 12,71 \pm 18,98 \quad 38,13 \pm 19,33] \\
& \mathrm{f}\left(\mathrm{x}_{\text {best }}\right)=\left[1534,1 \mathrm{dcm}^{3} \quad 336,72 \mathrm{~m} / \mathrm{s} \quad 243,36 \mathrm{~mm} \quad 4446,3 \mathrm{~mm}\right] \\
& \mathrm{f}\left(\mathrm{x}_{\mathrm{med}}\right)=\left[1128,86 \pm 405,2 \mathrm{dcm}^{3} \quad 306,54 \pm 30,17 \mathrm{~m} / \mathrm{s} \quad 184,56 \pm 58,8 \mathrm{~mm} \quad 4487,9 \pm 41,6 \mathrm{~mm}\right]
\end{aligned}
$$

Os resultados do Simulated Annealing tradicional foram semelhantes aos resultados apresentados acima, porém com um custo computacional muito mais elevado, na ordem de aproximadamente 100 vezes. Isso mais uma vez comprova a eficiência das mudanças propostas neste presente artigo. O número de avaliação da função objetivo ficou em torno de 100 a 120, sendo que o valor máximo de avaliação da função objetivo foi extrapolado para 20000. Já o número de temperaturas foi estipulado em 40 e o número de iterações em 3000.

Como se trata de um problema com vários mínimos locais houve a visitação de muitos pontos para obter o melhor, normalmente esse número é superior a 100.

Todos as restrições do problema foram obedecidas dentro de uma tolerância pré estabelecida para os resultados apresentados.

\section{AGRADECIMENTOS}

O primeiro autor agradece ao CNPQ pela bolsa de iniciação científica no processo IC-CNPq2013-EXA015.

\section{REFERÊNCIAS}

Arora, J. S., 1989, Introduction to Optimum Design, McGraw-Hill, Singapore

Bradão, M. A. L., Estudo de alguns métodos determinísticos de otimização irrestrita, Dissertação, Universidade Federal de Uberlândia.

Faria, E.D. e Saramago, S.F.P., 2001, Constraint optimization problems using simulated annealing, Ciência \& Engenharia, Brasil, V. 10, n. 1, p. 69-75. 
Saramago, S.F.P. e Souza, B.N., 2006, Projeto ótimo de uma caixa de transmissão de um torno, Encontro de Iniciação Científica, Universidade Federal de Uberlândia.

Saramago, S.F.P., 2003, Métodos de Otimização Randômica: Algoritmos Genéticos e Simulated Annealing, SBMAC, São Carlos, v.6. p.35.

Sartini, L. O., 2005, Uma Contibuição ao Estudo dos Métodos de Otimização Multi-Objetivo, Dissertação, Universidade Federal de Uberlândia.

Vanderplaats, G. N., 1999, Numerical Optimization Techniques for Engineering Design, Vanderplaats Research and Development, Inc., $3^{\text {rd }}$ ed.

\title{
RESPONSABILIDADE AUTORAL
}

\section{IMPROVED SIMULATED ANNEALING}

\author{
Vinícius Nunes Carvalho, viniciusncarvalho92@gmail.com ${ }^{1}$ \\ Sezimária de Fátima Pereira Saramago, saramago@ufu.br ${ }^{2}$ \\ ${ }^{1}$ Universidade Federal de Uberlândia - Faculdade de Engenharia Mecânica, Av. João Naves de Ávila, nº 2160, \\ Uberlândia, Minas Gerais, Brasil. \\ ${ }^{2}$ Universidade Federal de Uberlândia - Faculdade de Matemática, Av. João Naves de Ávila, no 2160, Uberlândia, \\ Minas Gerais, Brasil.
}

\begin{abstract}
The main objective of this work is to improve the algorithm known as Simulated Annealing, in order to avail their potentialities and increase their efficiency to solve optimization problems. In this research, it is intended to modify the basic algorithm of Simulated Annealing developing the method called Improved Simulated Annealing (ISA). The original algorithm is serial in its conception, and requires a high computational time. Each cycle of the algorithm consists of the generation, evaluation of the objective function and application of decision criteria. Several iterations for each temperature value are required, only at the end of this cycle the value of the optimum point is updated. The algorithm parameters are: a function cost that represents the energy of the system; the design variables that describe its configuration and the temperature, which is a control parameter. If the temperature parameter (T) has magnitude much higher than the standard deviation of the function in the range, almost all points are accepted. However, if $T$ is equal to zero the method becomes a random search for the minimum. Thus, it is adopted: $T_{i}$ as the standard deviation value of the objective function in the range studied and $T_{f}$ takes the desired order of magnitude for the accuracy of the optimum point. With this study it is intended that the efficiency of the method is improved by specific modifications made in the program seeking the best use of its capacity for the variables involved in the project. Besides, the algorithm is adapted to work with multi-objective functions in the presence of constraints. The developed algorithm is applied to the optimal design of a gearbox and the results presented to demonstrate the efficiency of the proposed methodology.
\end{abstract}

Keywords: Optimization, Simulated Annealing, Improved Simulated Annealing, Gearbox. 